\title{
Are blood pressure and diabetes additive or synergistic risk factors? Outcome in 8494 subjects randomly recruited from 10 populations
}

\author{
Thomas Sehestedt ${ }^{1}$, Tine W Hansen ${ }^{2}$, Yan $\mathrm{Li}^{3}$, Tom Richart ${ }^{4,5}$, Jose Boggia ${ }^{6}$, Masahiro Kikuya ${ }^{7}$, Lutgarde Thijs ${ }^{4}$, \\ Katarzyna Stolarz-Skrzypek ${ }^{8}$, Edoardo Casiglia ${ }^{9}$, Valérie Tikhonoff ${ }^{9}$, Sofia Malyutina ${ }^{10}$, Yuri Nikitin ${ }^{10}$, \\ Kristina Björklund-Bodegård ${ }^{11}$, Tatiana Kuznetsova ${ }^{4}$, Takayoshi Ohkubo ${ }^{7}$, Lars Lind ${ }^{11}$, Christian Torp-Pedersen ${ }^{1}$, \\ Jørgen Jeppesen ${ }^{1}$, Hans Ibsen ${ }^{1}$, Yutaka Imai ${ }^{7}$, Jiguang Wang ${ }^{3}$, Edgardo Sandoya ${ }^{12}$, Kalina Kawecka-Jaszcz ${ }^{8}$ \\ and Jan A Staessen ${ }^{4,5}$, on behalf of the International Database on Ambulatory Blood Pressure Monitoring \\ in Relation to Cardiovascular Outcomes (IDACO) Investigators
}

\begin{abstract}
It remains unknown whether diabetes and high blood pressure (BP) are simply additive risk factors for cardiovascular outcome or whether they act synergistically and potentiate one another. We performed 24-h ambulatory BP monitoring in 8494 subjects (mean age, 54.6 years; $47.0 \%$ women; $6.9 \%$ diabetic patients) enrolled in prospective population studies in 10 countries. In multivariable-adjusted Cox regression, we assessed the additive as opposed to the synergistic effects of BP and diabetes in relation to a composite cardiovascular endpoint by testing the significance of appropriate interaction terms. During 10.6 years (median follow-up), 1066 participants had a cardiovascular complication. Diabetes mellitus as well as the 24-h ambulatory BP were independent and powerful predictors of the composite cardiovascular endpoint. However, there was no synergistic interaction between diabetes and 24-h, daytime, or nighttime, systolic or diastolic ambulatory BP ( $P$ for interaction, $0.07 \leqslant P \leqslant 0.97)$. The only exception was a borderline synergistic effect between diabetes and daytime diastolic BP in relation to the composite cardiovascular endpoint $(P=0.04)$. In diabetic patients, with normotension as the reference group, the adjusted hazard ratios for the cardiovascular endpoint were 1.35 (95\% confidence interval $(\mathrm{Cl}), 0.87-2.11)$ for white-coat hypertension, $1.78(95 \% \mathrm{Cl}, 1.22-2.60)$ for masked hypertension and $2.44(95 \% \mathrm{Cl}, 1.92-3.11)$ for sustained hypertension. The hazard ratios for non-diabetic subjects were not different from those of diabetic patients ( $P$-values for interaction, $0.09 \leqslant P \leqslant 0.72$ ). In conclusion, in a large international population-based database, both diabetes mellitus and BP contributed equally to the risk of cardiovascular complications without evidence for a synergistic effect.
\end{abstract}

Hypertension Research (2011) 34, 714-721; doi:10.1038/hr.2011.6; published online 10 February 2011

Keywords: ambulatory blood pressure; cardiovascular risk factors; diabetes mellitus; epidemiology; population science

\section{INTRODUCTION}

Conventional blood pressure (BP) measurement is subject to substantial random fluctuations, partly because of measurement error and to the arousing effect of the observer on the individual, and partly because of biologic variability, such as circadian variation. Compared with conventional sphygmomanometry, ambulatory BP recordings have higher reproducibility, and therefore provide a better estimate of a subject's usual BP and cardiovascular prognosis. ${ }^{1-3}$ Because of impaired baroreflexes and stiffer arteries, ${ }^{4}$ patients with diabetes mellitus have higher BP variability ${ }^{5}$ and are more likely to be hypertensive than non-diabetic subjects. ${ }^{6,7}$

Notwithstanding the well-known advantages of ambulatory BP monitoring, few prospective studies of diabetic patients have applied this technique to examine the association between cardiovascular risk

\footnotetext{
${ }^{1}$ The Copenhagen University Hospital, Copenhagen, Denmark; ${ }^{2}$ The Research Center for Prevention and Health, and Department of Clinical Physiology, Nuclear Medicine and PET, Rigshospitalet, Copenhagen University Hospital, Faculty of Health Sciences, Copenhagen, Denmark; ${ }^{3}$ The Center for Epidemiological Studies and Clinical Trials, Ruijn Hospital, Shanghai Jiaotong University School of Medicine, Shanghai, China; ${ }^{4}$ The Studies Coordinating Centre, Division of Hypertension and Cardiovascular Rehabilitation, Department of Cardiovascular Diseases, University of Leuven, Leuven, Belgium; ${ }^{5}$ The Department of Epidemiology, Maastricht University, Maastricht, The Netherlands; ${ }^{6}$ The Departamento de Fisiopatología, Hospital de Clínicas, Universidad de la República, Montevideo, Uruguay; ${ }^{7}$ The Tohoku University Graduate School of Pharmaceutical Science and Medicine, Sendai, Japan; ${ }^{8}$ The First Department of Cardiology and Hypertension, Jagiellonian University Medical College, Kraków, Poland; ${ }^{9}$ The Department of Clinical and Experimental Medicine, University of Padova, Padova, Italy; ${ }^{10}$ The Institute of Internal Medicine, Novosibirsk, Russia; ${ }^{11}$ The Department of Public Health and Caring Sciences, Uppsala University, Uppsala, Sweden and ${ }^{12}$ The Asociación Espaňola Primera de Socorros Mutuos, Montevideo, Uruguay

Correspondence: Professor JA Staessen, Studies Coordinating Centre, Division of Hypertension and Cardiovascular Rehabilitation, Department of Cardiovascular Diseases, University of Leuven, Campus Sint Rafaël, Kapucijnenvoer 35, Block d, Box 7001, BE-3000 Leuven, Belgium.

E-mail: jan.staessen@med.kuleuven.be or ja.staessen@epid.unimaas.nl
}

Received 7 May 2010; revised 22 December 2010; accepted 27 December 2010; published online 10 February 2011 
and $\mathrm{BP}^{8}$ There is an abundant literature based on conventional BP measurement showing that the coexistence of hypertension and diabetes mellitus substantially enhances the risk of cardiovascular complications. ${ }^{9-11}$ However, it remains uncertain whether diabetes and hypertension are simply additive risk factors in relation to cardiovascular outcome or whether they act synergistically and potentiate one another. Also, the risk related to white-coat hypertension and masked hypertension in patients with diabetes compared with subjects without diabetes is not well known. To address these questions with great precision, we relied on the predictive power of ambulatory BP recordings, which exceeds that of conventional BP measurement. We analyzed randomly recruited population cohorts included in the large International Database on Ambulatory blood pressure monitoring in relation to Cardiovascular Outcomes (IDACOs).

\section{METHODS}

\section{Study population}

Studies were eligible for inclusion in the IDACO database if they involved a random population sample, if information about the conventional and ambulatory BP and cardiovascular risk factors were available at baseline, and if subsequent follow-up covered both fatal and non-fatal outcomes (for details see Thijs et al. ${ }^{12}$ ). For this study, we incorporated 10804 subjects from 10 centers. We excluded $2310(21.4 \%)$, because they were younger than 18 years at enrollment $(n=250)$, because their conventional BP had not been measured ( $n=220)$, or because there were fewer than 10 daytime $(n=146)$ or 5 nighttime readings ( $n=1983$ ). The number of subjects statistically analyzed totaled 8494 and included: 2142 residents from Copenhagen, Denmark; ${ }^{13} 1124$ from Noorderkempen, Belgium; ${ }^{14} 1317$ from Ohasama, Japan; ${ }^{15} 1438$ from Montevideo, Uruguay; ${ }^{16} 349$ from the JingNing county, China; ${ }^{17} 1097$ older men from Uppsala, Sweden, ${ }^{18} 165$ subjects from Pilsen, the Czech Republic; ${ }^{19} 308$ subjects from Kraków, Poland; ${ }^{19} 310$ subjects from Padova, Italy; ${ }^{19}$ and 244 subjects from Novosibirsk, the Russian Federation. ${ }^{19,20}$ Supplementary Table S1 of the Data Supplement published online lists the average number of daytime and nighttime readings by cohort and for all cohorts combined. All studies in the IDACO database received ethical approval, and all participants gave informed written consent.

\section{Measurements}

Conventional BP was measured by trained observers with a mercury sphygmomanometer, ${ }^{13,14,17-20}$ or with validated auscultatory ${ }^{21,22}$ (USM-700F, UEDA Electronic Works, Tokyo, Japan) ${ }^{15}$ or oscillometric (OMRON HEM-705CP, Omron Corporation, Tokyo, Japan ${ }^{16}$ devices, using the appropriate cuff size, with participants in the sitting ${ }^{13-17,19,20}$ or supine ${ }^{14}$ position. Conventional BP was the average of two consecutive readings obtained either at the person's home, ${ }^{14,16,17,19,20}$ or at an examination center. ${ }^{13,15,18}$ We used portable BP monitors to obtain readings every $30 \mathrm{~min}$ throughout the whole day, ${ }^{15}$ or at intervals ranging from 15 (see ref. 13) to $30 \mathrm{~min}^{18}$ during daytime and from 30 (see ref. 15) to $60 \mathrm{~min}^{13}$ at night. The devices used an auscultatory algorithm (Accutracker II, SunTech Medical Instruments Inc, Research Triangle Park, NC, USA) in Uppsala ${ }^{18}$ or an oscillometric technique (SpaceLabs 90202 and 90207, SpaceLabs Medical Inc., Redmond, WA, USA, and ABPM-630, Nippon Colin, Komaki, Japan) in the remaining cohorts. ${ }^{13-17,19,20}$

When accounting for the daily pattern of activities of the participants, we defined daytime as the interval ranging from 1000 to 2000 hours in Europeans ${ }^{13,14,19,20}$ and South Americans, ${ }^{16}$ and from 0800 to 1800 hours in Asians. ${ }^{15,17}$ The corresponding nighttime intervals ranged from 2400 to 0600 hours $^{13,14,16,18-20}$ and from 2200 to 0400 hours. ${ }^{15,17}$ These fixed-time intervals eliminate the transition periods in the morning and evening when $\mathrm{BP}$ changes rapidly, resulting in daytime and nighttime BP levels that are within $1-2 \mathrm{~mm} \mathrm{Hg}$ of the awake and asleep levels. ${ }^{17,23}$ Within individual subjects, we weighted the means of the ambulatory BP by the interval between readings.

White-coat hypertension was a raised conventional BP in the presence of a normal daytime BP and masked hypertension was an elevated ambulatory BP combined with a normal conventional BP. We defined conventional hypertension as a BP equal to or higher than $140 \mathrm{~mm} \mathrm{Hg}$ systolic or $90 \mathrm{~mm} \mathrm{Hg}$ diastolic. ${ }^{24}$ The corresponding thresholds for daytime ambulatory hypertension were $135 \mathrm{~mm} \mathrm{Hg}$ systolic and $85 \mathrm{~mm} \mathrm{Hg}$ diastolic. ${ }^{24}$ For categorical analyses based on these thresholds, patients on antihypertensive drug treatment were classified according to their treated BP.

At baseline, all subjects completed a questionnaire inquiring about medical history, intake of drugs, smoking and alcohol intake. Body mass index was body weight in kilograms divided by height in meters squared. Serum cholesterol and blood glucose concentrations were measured by automated enzymatic methods. Diabetes mellitus was the use of antidiabetic drugs, ${ }^{13-20} \mathrm{a}$ fasting blood glucose concentration of at least $7.0 \mathrm{mmoll}^{-1},{ }^{13-20}$ a random blood glucose concentration of at least $11.1 \mathrm{mmoll}^{-1},{ }^{14,15,17}$ a self-reported diagnosis ${ }^{14,16,17}$ or diabetes documented in practice or hospital records. ${ }^{16}$

\section{Ascertainment of events}

In each cohort, outcomes were validated against source documents described in previous publications. ${ }^{12,15,17,18,25,26}$ The validation process was the same in the Belgian study ${ }^{26}$ and in all other studies included in the European Project on Genes in Hypertension (Novosibirsk, Pilsen, Padova and Kraków). ${ }^{27}$

Fatal and non-fatal stroke (ICD8/9 430-434 and 436, ICD10 I60-I64 and I67-I68) did not include transient ischemic attacks. Coronary events included fatal ischemic heart disease (ICD8 411-412, ICD9 411 and 414, ICD10 I20 and I24-I25), sudden death (CD8 427.2 and 795, ICD9 427.5 and 798, ICD10 I46 and R96), non-fatal myocardial infarction (ICD8/9 410, ICD10 I21-I22), and coronary revascularization. Cardiac events encompassed coronary endpoints and fatal and non-fatal heart failure (ICD8 428 and 427.1-427.2 and 429.0, ICD9 429, ICD10 I50 and J81). Hospitalizations for unstable angina were coded as ischemic heart disease. In the Danish and Swedish cohorts, the diagnosis of heart failure required admission to the hospital. In the other cohorts, heart failure was either a clinical diagnosis or the diagnosis on the death certificate, but in all cases it was validated against hospital files or the records held by general practitioners. The composite cardiovascular end point included all aforementioned endpoints plus cardiovascular mortality (ICD8 390-448, ICD9 3900-4599, ICD10 I00-I79 and R96). In all outcome analyses, we only considered the first event within each category.

\section{Statistical methods}

For database management and statistical analysis, we used SAS software, version 9.1.3 (SAS Institute, Cary, NC, USA). For comparison of means and proportions, we applied the large sample z-test and the $\chi^{2}$-statistic, respectively. Statistical significance was a $\alpha$-level of 0.05 or less on two-sided tests. After stratification for cohort and sex, we interpolated missing values of body mass index $(n=12)$ and total serum cholesterol $(n=58)$ from the regression slope on age. In participants with unknown smoking habits $(n=34)$, drinking status ( $n=445$ in Uppsala and 170 in the nine other cohorts) or treatment status with antidiabetic drugs $(n=285)$, we set the design variable to the cohort- and sexspecific mean of the codes $(0.1)$.

We used Cox regression model to multivariable-adjusted risks associated with BP (treated as continuous or categorical variables), diabetes mellitus or blood glucose. The covariables adjusted for included cohort, sex, age, body mass index, smoking and drinking, history of cardiovascular disease, antihypertensive drug treatment and total serum cholesterol. We adjusted for cohort by introducing five design variables in the Cox models. The Asian cohorts from JingNing and Ohasama were pooled, as were the European cohorts from Novosibirsk, Pilsen, Padova and Kraków. For categorical analyses based on the conventional and ambulatory $\mathrm{BP}$, we presented the hazard ratios as floating absolute risks and calculated their standard errors as described by Easton et al. ${ }^{28}$ This approach allows the calculation of a $95 \%$ confidence interval (CI) for the relative risk in the reference group. We ascertained that the proportional hazard assumption underlying Cox regression was fulfilled by testing the interaction between follow-up time and the explanatory variables. We evaluated differences between hazard ratios associated with white coat, masked and sustained hypertension, using the TEST statement in the PROC PHREG procedure, as implemented in the SAS package. We evaluated the additive as opposed to synergistic effects of BP and diabetes on endpoints, using 
the appropriate interaction terms of BP with the presence of diabetes or blood glucose.

\section{RESULTS}

\section{Characteristics of participants}

The study population comprised 589 patients with diabetes and 7905 subjects without diabetes (Table 1). The diagnosis of diabetes mellitus rested on a fasting blood glucose concentration of $7.0 \mathrm{mmoll}^{-1}$ or higher in 297 subjects (50.4\%), a random blood glucose of at least $11.1 \mathrm{mmoll}^{-1}$ in $17(2.9 \%)$ and use of antidiabetic drugs in 194

Table 1 Baseline characteristics

\begin{tabular}{|c|c|c|}
\hline Characteristics & $\begin{array}{c}\text { No diabetes } \\
(\mathrm{n}=7905)\end{array}$ & $\begin{array}{l}\text { Diabetes } \\
(\mathrm{n}=589)\end{array}$ \\
\hline \multicolumn{3}{|l|}{ Anthropometrics } \\
\hline Female, $n(\%)$ & $3756(47.5)$ & $232(39.4)$ \\
\hline Mean age, years & $54.0 \pm 15.2$ & $63.3 \pm 10.1$ \\
\hline Mean body mass index, $\mathrm{kg} \mathrm{m}^{-2}$ & $25.4 \pm 4.1$ & $27.1 \pm 4.8$ \\
\hline Antihypertensive drug treatment, $n(\%)$ & $1566(19.8)$ & $252(42.8)$ \\
\hline \multicolumn{3}{|l|}{ Blood pressure } \\
\hline Hypertension, $n(\%)$ & $3373(42.7)$ & $388(65.9)$ \\
\hline Conventional systolic, $\mathrm{mm} \mathrm{Hg}$ & $130.8 \pm 20.3$ & $140.9 \pm 20.6$ \\
\hline Conventional diastolic, $\mathrm{mm} \mathrm{Hg}$ & $79.8 \pm 11.5$ & $81.1 \pm 12.4$ \\
\hline Daytime systolic, $\mathrm{mm} \mathrm{Hg}$ & $130.8 \pm 15.1$ & $136.3 \pm 16.3$ \\
\hline Daytime diastolic, $\mathrm{mm} \mathrm{Hg}$ & $78.8 \pm 9.2$ & $79.9 \pm 9.6$ \\
\hline Nighttime systolic, mm Hg & $112.2 \pm 15.0$ & $119.0 \pm 16.9$ \\
\hline Nighttime diastolic, $\mathrm{mm} \mathrm{Hg}$ & $64.6 \pm 9.2$ & $67.0 \pm 9.3$ \\
\hline 24-h systolic, $\mathrm{mm} \mathrm{Hg}$ & $123.8 \pm 14.1$ & $130.5 \pm 15.2$ \\
\hline 24-h diastolic, $\mathrm{mm} \mathrm{Hg}$ & $73.8 \pm 8.4$ & $75.5 \pm 8.5$ \\
\hline \multicolumn{3}{|l|}{ Other risk factors } \\
\hline Fasting blood glucose, $\mathrm{mmol}^{-1_{\mathrm{a}}}$ & $5.0 \pm 0.7$ & $8.7 \pm 2.9$ \\
\hline Current smokers, $n(\%)$ & $2318(29.4)$ & $122(20.9)$ \\
\hline Current drinkers, $n(\%)$ & $3880(52.5)$ & $233(47.6)$ \\
\hline Serum cholesterol, mmol I-1 \pm s.d. & $5.64 \pm 1.17$ & $5.65 \pm 1.13$ \\
\hline Previous cardiovascular disease, $n(\%)$ & $647(8.2)$ & $87(14.8)$ \\
\hline
\end{tabular}

Values are mean \pm s.d. or number of subjects (\%). Hypertension was a conventional blood pressure of at least $140 \mathrm{mmHg}$ systolic or $90 \mathrm{mmHg}$ diastolic or use of antihypertensive drugs. All between-group differences were significant $(P \leqslant 0.03$, with the exception of serum cholesterol $(P=0.85)$.

aFasting blood glucose was available in 6188 participants.
(32.9\%). The number of subjects with diabetes mellitus in the different cohorts was $73(3.4 \%)$ in Copenhagen, $232(17.6 \%)$ in Ohasama, $36(3.2 \%)$ in Nooderkempen, $121(11.3 \%)$ in Uppsala, 88 $(6.1 \%)$ in Montevideo, none in JingNing, $6(2.5 \%)$ in Novosibirsk, 8 (4.9\%) in Pilsen, 11 (3.6\%) in Padova and 14 (4.6\%) in Kraków.

Compared with non-diabetic subjects, patients with diabetes were older, had a slightly higher body mass index, but similar total serum cholesterol (Table 1). The diabetic group had higher BP, regardless of whether the conventional or the 24-h BPs were analyzed. Antihypertensive treatment and a history of previous cardiovascular disease were more frequent among diabetic patients, whereas the opposite was the case for the prevalence of smoking. The baseline characteristics of the European (Supplementary Table S1) and the Asian and South American (Supplementary Table S2) cohorts and the incidence rates of the events in these cohorts (Supplementary Tables S3 and S4) are presented in the online Data Supplement.

\section{Risk associated with diabetes mellitus and the 24-h BP}

In the overall study population, median follow-up was 10.6 years (5th to 95 th percentile, 2.5-15.4). Across cohorts, median follow-up ranged from 2.5 years $(2.3-2.6)$ in JingNing to 13.1 years (1.1-15.7) in Noorderkempen. A total of 1215 participants died (14.3 per 1000 person-years) and 1066 had a fatal or non-fatal cardiovascular complication (13.1 per 1000 person-years). With adjustments applied for cohort, sex, age, body mass index, 24-h systolic or diastolic BP, smoking and drinking, treatment for hypertension, history of cardiovascular disease and total serum cholesterol, diabetes mellitus was an independent and powerful predictor of outcome (Table 2). Similarly, the 24-h systolic and diastolic BPs were powerful predictors of outcome with diabetes mellitus and other covariables in the model (Table 2).

\section{Comparison of risk estimates between subjects with and without diabetes}

With adjustments applied as before, Table 3 list the standardized hazard ratios for mortality and cardiovascular events in relation to systolic and diastolic BPs, separately in non-diabetic subjects and patients with diabetes. The hazard ratios for diabetes and BP were additive, irrespective of whether the conventional or the 24-h, daytime or nighttime BPs were considered. The $P$-values for a synergistic effect between diabetes and BP, tested by introducing the appropriate interaction terms in the Cox models, were not significant $(P$ for

Table 2 Multivariable adjusted standardized hazard ratios associated with diabetes mellitus and the 24-h blood pressure

\begin{tabular}{|c|c|c|c|c|c|c|}
\hline \multirow[b]{2}{*}{ Label } & \multicolumn{2}{|c|}{ Mortality } & \multicolumn{4}{|c|}{ Fatal and non-fatal cardiovascular outcomes } \\
\hline & Total & Cardiovascular & Composite & Stroke & Cardiac & Coronary \\
\hline Events, $n$ & 1215 & 471 & 1066 & 452 & 604 & 436 \\
\hline Number of person-years & 84864 & 84864 & 81542 & 83288 & 83034 & 83602 \\
\hline \multicolumn{7}{|l|}{ Diabetes } \\
\hline Model S & $1.38(1.16-1.63)^{\ddagger}$ & $1.57(1.22-2.01)^{\ddagger}$ & $1.43(1.20-1.70)^{\ddagger}$ & $1.39(1.08-1.80)^{*}$ & $1.54(1.21-1.95)^{\ddagger}$ & $1.47(1.11-1.95)^{\dagger}$ \\
\hline Model D & $1.41(1.19-1.66)^{\ddagger}$ & $1.65(1.29-2.12)^{\ddagger}$ & $1.51(1.27-1.80)^{\ddagger}$ & $1.48(1.15-1.91)^{\dagger}$ & $1.62(1.28-2.06)^{\ddagger}$ & $1.54(1.16-2.05)^{\dagger}$ \\
\hline \multicolumn{7}{|l|}{ Blood pressure } \\
\hline 24-h systolic (s.d.=14.27 mm Hg) & $1.14(1.08-1.21)^{\ddagger}$ & $1.36(1.25-1.48)^{\ddagger}$ & $1.38(1.30-1.45)^{\ddagger}$ & $1.54(1.42-1.68)^{\ddagger}$ & $1.29(1.20-1.39)^{\ddagger}$ & $1.23(1.13-1.35)^{\ddagger}$ \\
\hline 24-h diastolic (s.d. $=8.46 \mathrm{~mm} \mathrm{Hg}$ ) & $1.12(1.05-1.19)^{\ddagger}$ & $1.32(1.21-1.45)^{\ddagger}$ & $1.31(1.23-1.39)^{\ddagger}$ & $1.46(1.33-1.61)^{\ddagger}$ & $1.22(1.12-1.32)^{\ddagger}$ & $1.21(1.10-1.33)^{\ddagger}$ \\
\hline
\end{tabular}

The hazard ratios (95\% confidence interval) express the risk associated with diabetes mellitus or with one s.d. increase in the 24-h systolic or diastolic blood pressures. All models were adjusted for cohort, sex, age, body mass index, smoking and drinking, antihypertensive drug treatment, history of cardiovascular disease and total serum cholesterol. Models S/D also include the 24-h systolic/ diastolic blood pressure. Models for the $24-\mathrm{h}$ ambulatory blood pressure also included a design variable coding $(0,1)$ for the presence of diabetes mellitus. Significance of the hazard ratios: $\dagger P<0.01, \ddagger P<0.001$. 
Table 3 Multivariable adjusted standardized hazard ratios associated with blood pressure in non-diabetic subjects and diabetic patients

\begin{tabular}{|c|c|c|c|c|c|c|c|}
\hline \multirow[b]{2}{*}{ Label } & & \multicolumn{2}{|c|}{ Mortality } & \multicolumn{4}{|c|}{ Fatal and non-fatal cardiovascular outcomes } \\
\hline & & Total & Cardiovascular & Composite & Stroke & Cardiac & Coronary \\
\hline \multirow[t]{3}{*}{ Number of person-years } & & 78834 & 78834 & 75982 & 77539 & 77207 & 77695 \\
\hline & $+\mathrm{DM}$ & $167(28.4)$ & $81(13.8)$ & $161(27.3)$ & 76 (12.9) & $87(14.8)$ & $61(10.4)$ \\
\hline & & 6030 & 6030 & 5560 & 5748 & 5827 & 5906 \\
\hline
\end{tabular}

Conventional

Systolic (s.d.=20.53 mm Hg) $\quad-$ DM $1.10(1.03-1.18)^{\dagger} 1.33(1.20-1.48)^{\ddagger} 1.25(1.16-1.34)^{\ddagger} 1.38(1.24-1.54)^{\ddagger} 1.17(1.07-1.29)^{\ddagger} 1.14(1.02-1.27)^{*}$ +DM $1.20(1.2-1.42)^{*} \quad 1.29(1.02-1.64)^{*} 1.29(1.10-1.52)^{\dagger} 1.54(1.21-1.96)^{\ddagger} 1.19(0.95-1.48) \quad 1.03(0.79-1.35)$

Diastolic (s.d. $=11.58 \mathrm{~mm} \mathrm{Hg}$ ) -DM $1.02(0.96-1.10) \quad 1.18(1.06-1.31)^{\dagger} 1.17(1.09-1.26)^{\ddagger} 1.27(1.14-1.42)^{\ddagger} 1.11(1.01-1.21)^{*} 1.12(1.00-1.25)^{*}$ +DM $1.16(0.98-1.37) \quad 1.20(0.94-1.53) \quad 1.22(1.03-1.44)^{*} 1.29(1.02-1.64)^{*} 1.17(0.92-1.47) \quad 1.08(0.81-1.43)$

Ambulatory

24-h systolic (s.d. $=14.26 \mathrm{~mm} \mathrm{Hg}) \quad-$ DM $1.13(1.06-1.20)^{\ddagger} 1.35(1.23-1.48)^{\ddagger} \quad 1.36(1.28-1.44)^{\ddagger} 1.50(1.37-1.65)^{\ddagger} \quad 1.28(1.18-1.39)^{\ddagger} \quad 1.24(1.12-1.37)^{\ddagger}$ +DM $1.21(1.04-1.40)^{*} 1.40(1.14-1.72)^{\dagger} 1.46(1.26-1.68)^{\ddagger} 1.82(1.46-2.27)^{\ddagger} 1.30(1.06-1.59)^{\dagger} 1.19(0.94-1.51)$

24-h diastolic (s.d. $=8.46 \mathrm{~mm} \mathrm{Hg}$ ) -DM $1.10(1.03-1.17)^{\dagger} 1.29(1.17-1.43)^{\ddagger} 1.29(1.21-1.38)^{\ddagger} 1.44(1.30-1.59)^{\ddagger} 1.20(1.10-1.31)^{\ddagger} 1.19(1.08-1.32)^{\ddagger}$ +DM $1.25(1.06-1.47)^{\dagger} 1.48(1.18-1.85)^{\ddagger} 1.44(1.22-1.69)^{\ddagger} 1.63(1.29-2.07)^{\ddagger} 1.29(1.03-1.62)^{*} 1.33(1.01-1.74)^{*}$

Daytime systolic (s.d.=15.24 mm Hg) $\quad-$ DM $1.07(1.01-1.14)^{*} 1.28(1.16-1.42)^{\ddagger} 1.29(1.21-1.38)^{\ddagger} 1.43(1.29-1.57)^{\ddagger} \quad 1.24(1.14-1.35)^{\ddagger} 1.25(1.13-1.38)^{\ddagger}$ +DM $1.12(0.96-1.31) \quad 1.26(1.01-1.56)^{*} 1.43(1.23-1.65)^{\ddagger} 1.66(1.33-2.08)^{\ddagger} 1.29(1.05-1.59)^{*} 1.16(0.90-1.48)$

Daytime diastolic (s.d.=9.21 mm Hg) $\quad$-DM $1.05(0.98-1.12) \quad 1.21(1.09-1.34)^{\ddagger} 1.21(1.14-1.30)^{\ddagger} 1.35(1.22-1.49)^{\ddagger} \quad 1.14(1.04-1.24)^{\dagger} 1.17(1.05-1.29)^{\dagger}$ +DM $1.16(0.99-1.35) \quad 1.26(1.01-1.57)^{*} 1.38(1.18-1.62)^{\ddagger} 1.49(1.18-1.87)^{\ddagger} 1.27(1.02-1.57)^{*} 1.24(0.96-1.61)$

Nighttime systolic (s.d.=15.26 mm Hg) -DM $1.16(1.10-1.23)^{\ddagger} 1.31(1.20-1.42)^{\ddagger} 1.30(1.23-1.37)^{\ddagger} 1.39(1.28-1.50)^{\ddagger} 1.24(1.15-1.33)^{\ddagger} 1.16(1.06-1.27)^{\ddagger}$ +DM $1.23(1.08-1.41)^{\dagger} 1.36(1.14-1.63)^{\ddagger} 1.34(1.17-1.53)^{\ddagger} 1.67(1.36-2.05)^{\ddagger} 1.21(1.01-1.45)^{*} 1.17(0.95-1.46)$

Nighttime diastolic (s.d.=9.24 mm Hg) $\quad-$ DM $1.15(1.09-1.23)^{\ddagger} 1.30(1.18-1.43)^{\ddagger} 1.27(1.20-1.36)^{\ddagger} 1.39(1.26-1.54)^{\ddagger} \quad 1.19(1.10-1.29)^{\ddagger} 1.15(1.04-1.26)^{\ddagger}$ +DM $1.30(1.11-1.53)^{\dagger} 1.52(1.22-1.90)^{\ddagger} 1.32(1.12-1.55)^{\ddagger} 1.54(1.21-1.96)^{\ddagger} 1.16(0.93-1.45) \quad 1.27(0.99-1.64)$

The hazard ratios (95\% confidence intervals) express the risk associated with a one s.d. increase in blood pressure and were adjusted for cohort, sex, age, body mass index, smoking and drinking, antihypertensive drug treatment, history of cardiovascular disease and total serum cholesterol. $-/+$ DM: participants without/with diabetes. Significance of the hazard ratios: ${ }^{*} P<0.05,{ }^{\dagger} P<0.01$ and $\ddagger P<0.001$. There was no synergistic effect between diabetes mellitus and blood pressure ( $P$ for interaction $0.07 \leqslant P \leqslant 0.97$ ), with exception of daytime diastolic pressure in relation to the composite cardiovascular end point $(P=0.04)$.

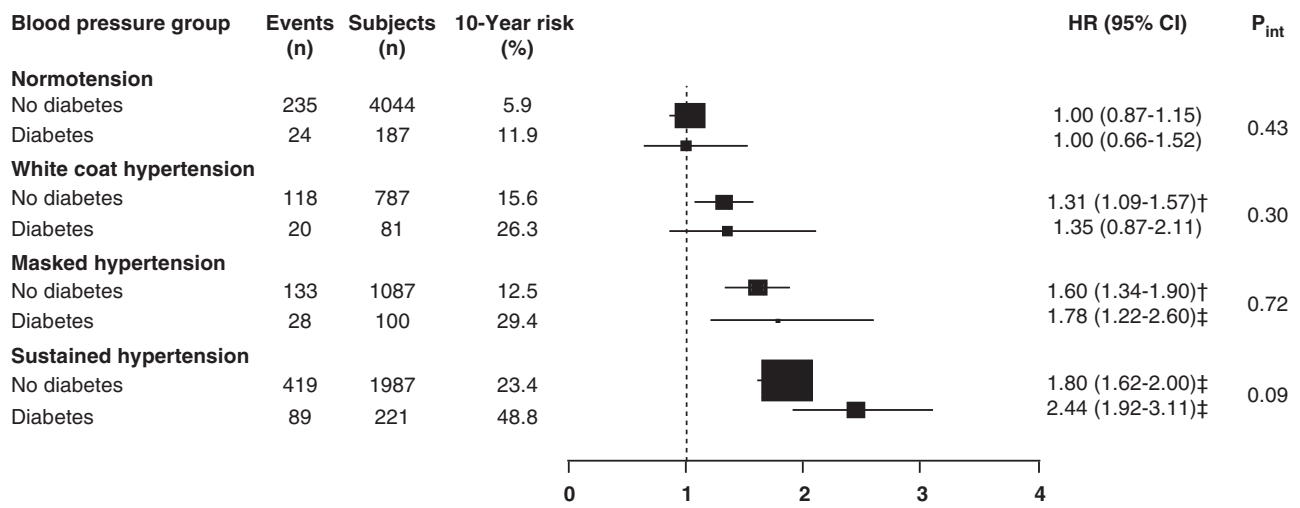

Figure 1 Floating hazard ratios for the composite cardiovascular endpoint in subjects without or with diabetes and with normotension, white-coat hypertension, masked hypertension or sustained hypertension. Normotension was the reference group (conventional blood pressure (CBP) $<140 / 90 \mathrm{~mm} \mathrm{Hg}$ and daytime ambulatory blood pressure $(\mathrm{dABP})<135 / 85 \mathrm{~mm} \mathrm{Hg}$ ). Hazard ratios are given for white-coat hypertension (CBP $\geqslant 140 / 90 \mathrm{~mm} \mathrm{Hg}$ and $\mathrm{dABP}$ $<135 / 85 \mathrm{~mm} \mathrm{Hg}$ ), masked hypertension (CBP $<140 / 90 \mathrm{~mm} \mathrm{Hg}$ and $\mathrm{dABP} \geqslant 135 / 85 \mathrm{~mm} \mathrm{Hg}$ ) and sustained hypertension (CBP $\geqslant 140 / 90 \mathrm{~mm} \mathrm{Hg}$ and $\mathrm{dABP}$ $\geqslant 135 / 85 \mathrm{~mm} \mathrm{Hg}$ ). Squares are proportional to the number of events per group. Horizontal lines denote the $95 \%$ confidence interval. Significance of the hazard ratios: ${ }^{\dagger} P<0.01, \ddagger P<0.001$. All analyses were adjusted for cohort, sex, age, body mass index, smoking and drinking, antihypertensive drug treatment, history of cardiovascular disease and total serum cholesterol.

interaction, $0.07 \leqslant P \leqslant 0.97)$. The only exception with borderline significance was the synergistic effect between diabetes mellitus and the daytime diastolic BP in relation to the composite cardiovascular end point ( $P$ for interaction, 0.04).

Categorical analyses confirmed the results in Table 3. Figure 1 shows the hazards ratios associated with normotension, white-coat, masked and sustained hypertension for the composite cardiovascular endpoint separately in non-diabetic subjects and in patients with diabetic mellitus. Cardiovascular risk increased across the hypertensive groups. Masked hypertension and sustained hypertension were associated with a significantly higher risk of a composite cardiovascular event, compared with normotension. White-coat hypertension, compared 


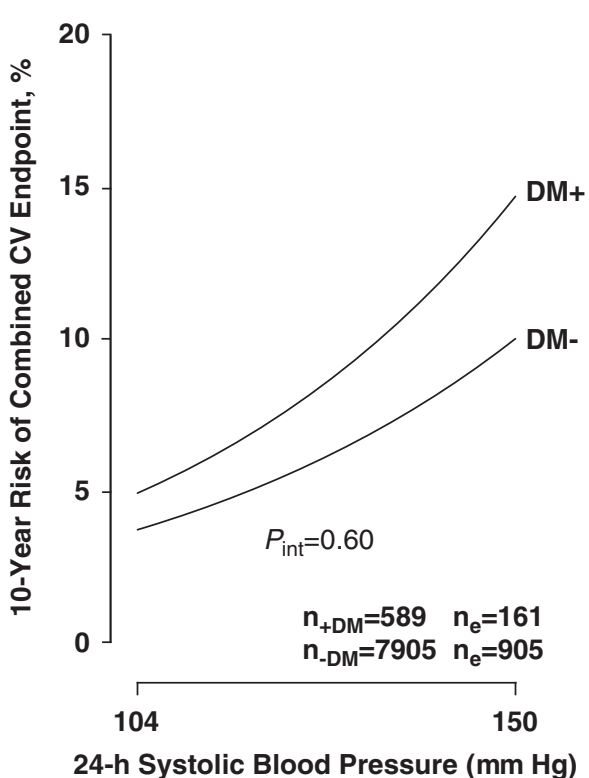

b

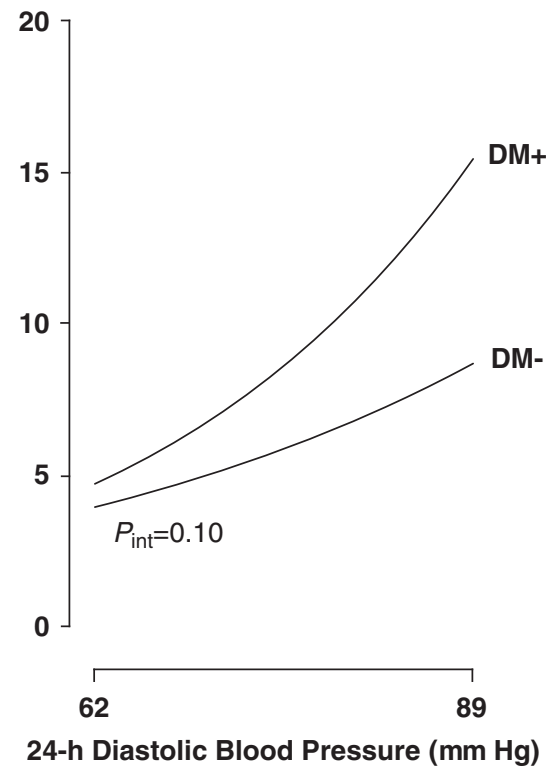

Figure 2 10-year absolute risk of the composite cardiovascular end point associated with the 24-h systolic (a) and diastolic (b) blood pressures in patients with diabetes and non-diabetic subjects. The analysis was standardized to the distributions (mean or ratio) of cohort, age, sex, body mass index, smoking and drinking, treatment with antihypertensive drugs, history of cardiovascular disease and total serum cholesterol, and included an interaction term for diabetes and blood pressure. The risk functions span the 5th to 95th percentile interval of the 24-h blood pressure. +DM and -DM indicate diabetic and non-diabetic subjects, respectively, $e_{+D M} / n_{+D M}$ and $e_{-D M} / n_{-D M}$ denote the number of events and the number of subjects at risk in the $+D M$ and $-D M$ groups, respectively.

with normotension, was associated with a significantly higher risk in non-diabetic subjects $(P=0.004)$, but not in patients with diabetes $(P=0.18)$. The risk ratios for the non-diabetic subjects and the diabetic patients were not significantly different ( $P$-values for interaction indicating a synergistic effect, $0.09 \leqslant P \leqslant 0.72$ ).

Figure 2 shows the 10-year absolute risk associated with 24-h systolic (A) and diastolic (B) BP standardized to the distributions (means or ratios) of cohort, sex, age, body mass index, smoking and drinking, treatment with antihypertensive drugs, history of cardiovascular disease and total serum cholesterol, and included an interaction term for diabetes and BP. The significance of the interaction term was 0.60 for systolic BP and 0.10 for diastolic BP. Supplementary Figure S1 illustrates the 10-year risk of the composite cardiovascular endpoints by categories of BP and the presence or absence of a history of diabetes mellitus.

\section{Sensitivity analysis}

Information on fasting blood glucose was available in 6188 subjects (72.9\%): 2139 (99.9\%) from Copenhagen, 2 (0.2\%) from Ohasama, 507 (45.1\%) from Nooderkempen, 1097 (100\%) from Uppsala, 1118 (77.7\%) from Montevideo, 331 (95.5\%) from JingNing, 237 (97.1\%) from Novosibirsk, 157 (95.1\%) from Pilsen, 306 (98.7\%) from Padova and $294(95.5 \%)$ from Kraków. The 10-year absolute risks of a composite cardiovascular event associated with fasting blood glucose at different levels of 24-h systolic (A) or diastolic (B) BP appear in Figure 3. These analyses were adjusted for cohort, sex, age, body mass index, smoking and drinking, treatment with antidiabetic or antihypertensive drugs, history of cardiovascular disease and total serum cholesterol, and included an interaction term for blood glucose and BP. There was a borderline significant interaction between fasting blood glucose and 24-h systolic $(P=0.02)$, but not 24 -h diastolic BP $(P=0.38)$. As illustrated in Figure $3 \mathrm{a}$, the risk associated with higher 24-h systolic BP tended to increase somewhat less in the highest quintile of blood glucose. Supplementary Figure S2 illustrates the 10-year risk of the composite cardiovascular endpoints by categories of BP and blood glucose concentration. In addition, sensitivity analyses excluding the Uppsala cohort, ${ }^{18}$ in which conventional BP was measured in the supine instead of the standing position and in which the ambulatory BP was recorded by an auscultatory instead of an oscillometric technique, were confirmatory (Supplementary Data Table S5).

\section{DISCUSSION}

The principal finding of our study was that both diabetes mellitus and BP independently contributed to the risk of death or cardiovascular events. Both risk factors were additive with little evidence of a synergistic effect. To our knowledge, our study is the first to examine on a large scale the possible synergistic effects of $\mathrm{BP}$ and diabetes on health outcomes by means of ambulatory BP measurements. Only one other prospective study ${ }^{29}$ made use of ambulatory BP monitoring to examine the risk associated with BP in diabetic and non-diabetic patients. Eguchi et al. recruited 1268 patients from nine sites in Japan. ${ }^{29}$ All patients were referred for hypertension and $301(23.7 \%)$ had type- 2 diabetes. The mean age was 70.4 years. The patients were followed for a mean of 50 months and 100 cardiovascular events occurred. With adjustments applied for site, sex, age, clinic systolic BP, body mass index, antihypertensive drugs and serum creatinine, the hazard ratios associated with a $10 \mathrm{~mm} \mathrm{Hg}$ increment in the 24-h systolic BP were 1.44 (95\% CI, 1.15-1.80) in diabetic patients and 1.32 (95\% CI, 1.10-1.58) in non-diabetic subjects. In keeping with our current observations, there was no significant interaction between the presence of diabetes and the $24-\mathrm{h}$ systolic BP. ${ }^{29}$ Several studies, ${ }^{30-32}$ including a previous report from the IDACO consortium, ${ }^{3}$ confirmed that the ambulatory BP is a better predictor of cardiovascular risk than 

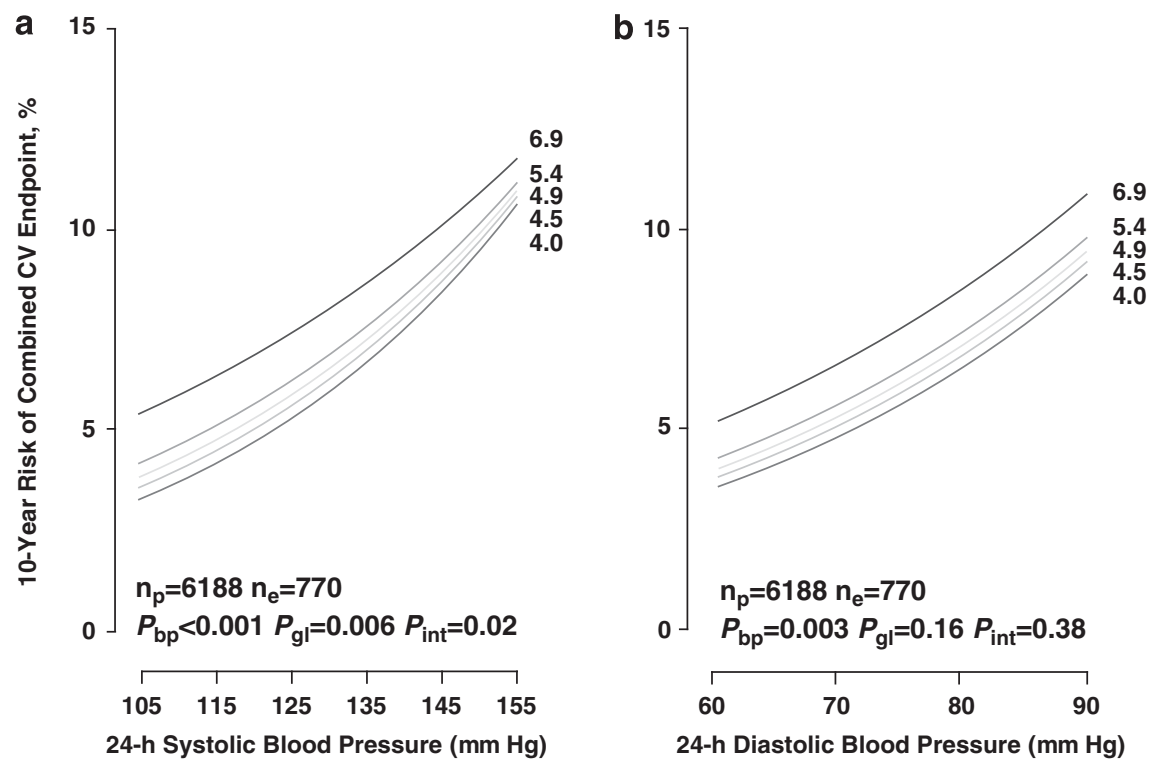

Figure 3 10-year absolute risk of the composite cardiovascular events associated with fasting blood glucose at different levels of 24-h systolic (a) or diastolic (b) blood pressure. The analyses were standardized to the distributions (mean or ratio) of cohort, sex, age, body mass index, smoking and drinking, treatment with antidiabetic or antihypertensive drugs, history of cardiovascular disease and total serum cholesterol, and included an interaction term for blood glucose and blood pressure. The risk functions span the 5th to 95th percentile interval of the 24-h blood pressure and correspond to the 5th, 25th, 50th, 75th and 95th percentiles of blood glucose. The ne and $\mathrm{np}$ indicate the number of events and participants at risk. Pgl and Pbp denote the significance of the independent contributions of fasting blood glucose and 24-h blood pressure. Pint denotes the significance of the interaction between fasting blood glucose and 24-h blood pressure.

the conventional BP, irrespective of whether the study participants had or did not have diabetes.

A number of prospective studies assessed the cardiovascular risk associated with BP in diabetic patients and non-diabetic subjects, but used conventional BP measurement. In the Multiple Risk Factor Intervention Trial, ${ }^{33}$ men aged $35-57$ years with no previous hospitalization for myocardial infarction and including 5163 diabetic patients and 342815 men without diabetes were followed up for 12 years. Diabetes was the use of antidiabetic drugs, but this report ${ }^{33}$ did not include any measurement of glucose in blood or urine. Cardiovascular mortality was three times higher in diabetic compared with non-diabetic men. BP was measured by auscultation and the average of the second and third readings was used in the analysis. The cardiovascular mortality was positively related to systolic BP $(P<0.001)$. From systolic BP levels below $120 \mathrm{~mm} \mathrm{Hg}$ to above $200 \mathrm{~mm} \mathrm{Hg}$, in diabetic men, cardiovascular mortality increased from 53.6 to 242.6 deaths per 10000 person-years, whereas in nondiabetic men mortality increased only from 12.2 to 121.7 deaths per 10000 person-years. Cardiovascular mortality increased in a steeper manner with BP in diabetic than in non-diabetic men. However, the hazard ratios associated with a $20 \mathrm{~mm} \mathrm{Hg}$ higher systolic BP, adjusted for race, age, income, total serum cholesterol and smoking was significantly higher for men without diabetes than men with diabetes (1.60 vs. $1.41 ; P=0.002)$, suggesting a significant interaction between $\mathrm{BP}$ and diabetes, but in the opposite direction than expected.

A meta-analysis by the Asia Pacific Cohort Studies Collaboration ${ }^{34}$ included 364566 subjects of whom 6\% had diabetes. Follow-up lasted 8 years. Diabetes was a self-reported diagnosis or based on measurement of blood glucose. In an analysis, only adjusted for age, there was a significant interaction between diabetes and systolic BP in relation to mortality from coronary heart disease among men, but not in women. The hazard ratio associated with a $10-\mathrm{mm} \mathrm{Hg}$ increase in systolic BP was 1.17 (95\% CI 1.13-1.23) in non-diabetic men and 1.02 (95\% CI
0.90-1.13) in diabetic men ( $P$ for difference, 0.011). In women, corresponding estimates were $1.27(95 \%$ CI $1.20-1.33)$ and 1.33 (95\% CI 1.15-1.52), respectively $(P=0.50) .{ }^{34}$ Iso et al. ${ }^{35}$ investigated 10582 Japanese, of whom 267 had type-2 diabetes. Follow-up lasted 17 years. The diagnosis of diabetes was based on the measurement of blood glucose or the use of antidiabetic medications. Hypertension was a BP equal to or higher than $160 \mathrm{~mm} \mathrm{Hg}$ systolic or $95 \mathrm{~mm} \mathrm{Hg}$ diastolic. Adjusted analyses revealed a significant interaction ( $P=0.006)$ between hypertension and diabetes in relation to the risk of non-ischemic stroke. The relative risks associated with diabetes were 3.1 (95\% CI, 1.8-5.5) and $1.2(95 \%$ CI, 0.7-2.3) in normotensive subjects and hypertensive patients, respectively. ${ }^{35}$ In contrast to our study, several of the previously mentioned prospective studies ${ }^{33-35}$ did not fully account for potential confounders, in particular the use of antihypertensive medications.

Other studies failed to demonstrate a significant interaction between diabetes mellitus or $\mathrm{HbA}_{1 \mathrm{c}}$ and $\mathrm{BP}$ in the prediction of cardiovascular death, ${ }^{36}$ a composite cardiovascular endpoint, $8,37-40$ stroke, ${ }^{40}$ or coronary heart disease. ${ }^{36,37}$ The sample size in these studies ranged from 4320 (see ref. 37) to 49582 (see ref. 40) participants and the prevalence of diabetes varied from 3.5 (see ref. 36) to $100 \%{ }^{37}$ The covariables accounted for in the analyses included ethnicity, ${ }^{37,39}$ sex, ${ }^{36-40}$ age, ${ }^{36-40}$ body mass index, ${ }^{36,39,40}$ smoking, ${ }^{36-38,40}$ alcohol intake, ${ }^{40}$ physical activity, ${ }^{40}$ education, ${ }^{40}$ history of cardiovascular disease, ${ }^{36,39}$ serum cholesterol, ${ }^{36,37,39,40}$ or albuminuria. $^{37}$

Masked hypertension was associated with increased risk both in non-diabetic subjects and in patients with diabetes, without any significant interaction. A major advantage of ambulatory BP monitoring is the ability to detect an elevated BP not identified by conventional BP measurements. Masked hypertension was present in $14.0 \%$ of our population and the increased risk of these individuals could not have been picked up without the use of ambulatory BP 
monitoring. This emphasizes the key role of ambulatory BP monitoring in clinical care.

Several risk prediction tools account of diabetes mellitus. The Systematic Coronary Risk Evaluation (SCORE) ${ }^{41}$ does not include diabetes, but considers the risk associated with diabetes as additive with that of other risk factors. Indeed, for any combination of risk factors, the SCORE estimate of risk is multiplied by two in diabetic men and multiplied by four in diabetic women. The Framingham score $^{42}$ and the Prospective Cardiovascular Münster score ${ }^{43}$ also treat the risk of diabetes as additive by adding a constant to the score provided by other risk factors. For the Framingham score, the constant is four for women and two for men, and for Prospective Cardiovascular Münster, the constant is six for either sex. Another approach, such as for instance implemented in the United Kingdom Diabetes Prospective Study risk engine, ${ }^{44}$ has been to develop specific risk scores in large diabetic cohorts. However, the prognostic superiority of these scores compared with for instance the Framingham score remains a matter of controversy. ${ }^{34,45,46}$

Whether the increased absolute risk associated with diabetes and BP justifies a lower BP target remains a matter of debate. In the recent Action to Control Cardiovascular Risk in Diabetes BP trial, ${ }^{47}$ patients with type 2 diabetes were randomized to a goal systolic BP below $140 \mathrm{~mm} \mathrm{Hg}$ (standard therapy group) or below $120 \mathrm{~mm} \mathrm{Hg}$ (intensive therapy group). After 4.7 years of follow-up, there were no significant differences between the two groups in a composite endpoint, consisting of cardiovascular death, non-fatal myocardial infarction and nonfatal stroke. Moreover, in line with our current results, there was no significant interaction between $\mathrm{HbA1c}$ and $\mathrm{BP}$ category in relation to the risk of the composite cardiovascular endpoint.

The strong points of our current report are the use of ambulatory BP monitoring instead of office measurement of BP, the large sample size representing populations from Europe, Asia and South America, and the large number of events, which occurred over a median followup of over 10 years. Our main analysis with diabetes as a categorical variable was supported by a continuous analysis in which we considered fasting blood glucose as an index of risk. Using continuous values of BP as well as blood glucose addresses the interaction with higher sensitivity than using arbitrary categories and avoids the problem of multiple testing across a large number of categories. On the other hand, our study also has some limitations. The analysis rested on 10 population-based cohorts with an overrepresentation of European subjects and might not be representative for other ethnic groups, in particular Blacks. Furthermore, anthropometric characteristics differed between cohorts and ambulatory BP monitoring was not standardized in terms of device type and intervals between successive readings. However, using a single SAS program ensured that daytime and nighttime periods were always defined in the same manner, using short, fixed clock-time intervals. All ambulatory BP means were weighted for the interval between successive readings. ${ }^{23}$ The definition of diabetes varied across cohorts. However, the sensitivity analyses in which we introduced blood glucose as a continuous variable were consistent with those, in which we treated diabetes mellitus as a categorical variable.

In conclusion, our study confirmed that BP was an important risk factor for cardiovascular mortality and morbidity among diabetic patients. We could not confirm the commonly held assumption that the risks of diabetes mellitus and BP are synergistic and not merely additive. This suggests that the same operational thresholds for the ambulatory BP might be applicable in diabetic patients and nondiabetic subjects. Nonetheless, because of the higher absolute risk among diabetic patients, awareness, treatment and control of hypertension in diabetic patients will translate into a larger absolute reduction of cardiovascular morbidity and mortality than in non-diabetic subjects.

\section{ACKNOWLEDGEMENTS}

The European Society of Hypertension supported the work of Thomas Sehestedt. The European Union (grants LSHM-CT-2006-037093 InGenious HyperCare and HEALTH-F4-2007-201550 HyperGenes), the Fonds voor Wetenschappelijk Onderzoek Vlaanderen, Ministry of the Flemish Community, Brussels, Belgium (grants G.0575.06 and G.0734.09) and the Katholieke Universiteit Leuven, Belgium (grants OT/00/25 and OT/05/49) gave support to the Studies Coordinating Centre. The Danish Heart Foundation (grant 01-2-9-9A-22914), the Beckett Fonden and the Lundbeck Fonden (grant R32-A2740) supported the studies in Copenhagen. The Bilateral Scientific and Technological Collaboration between China and Flanders, Ministry of the Flemish Community, Brussels (grant BIL02/10) supported the fellowship of Y Li in Leuven. The Ministries of Education, Culture, Sports, Science and Technology (grants 15790293, 16590433, 17790381, 18390192, 18590587, 19590929, 19790423, 20590629, 21390201 and 21591016) and of Health, Labor and Welfare (grants H17-Kenkou-007, H18-Junkankitou (Seishuu)-Ippan-012 and H20-Junkankitou (Seishuu)-Ippan-009, 013, Health Science Research Grants and Medical Technology Evaluation Research Grants), Grant-in-Aid for Japan Society for the Promotion of Science (JSPS) fellows (16.54041, 18.54042, 19.7152, 20.7198, 20.7477 and 20.54043), the Japan Atherosclerosis Prevention Fund, the Uehara Memorial Foundation, the Takeda Medical Research Foundation supported research in Japan, National Cardiovascular Research Grants and Biomedical Innovation Grants. The National Natural Science Foundation of China (grants 30871360 and 30871081), Beijing, China, and the Shanghai Commissions of Science and Technology (grant 07JC14047 and the "Rising Star" program 06QA14043) and Education (grant 07ZZ32 and the "Dawn" project 08SG20) supported the JingNing study in China. The funding organizations had no role in the design or conduct of the study; collection, management, analysis, or interpretation of the data; or preparation, review, or approval of the manuscript. The authors gratefully acknowledge the expert assistance of Ms. Sandra Covens and Ms. Ya Zhu (Studies Coordinating Centre, Division of Hypertension and Cardiovascular Rehabilitation, Department of Cardiovascular Diseases, University of Leuven, Leuven, Belgium).

Authors' Contributions: Dr Staessen had full access to all of the data and takes responsibility for the integrity of the data and the accuracy of the data analysis. Study concept and design: Staessen. Acquisition of the data: Sehestedt, Hansen, Li, Richart, Boggia, Kikuya, Thijs, Stolarz-Skrzypek, Casiglia, Tikhonoff, Malyutina, Nikitin, Björklund-Bodegård, Kuznetsova, Ohkubo, Lind, Torp-Pedersen, Jeppesen, Ibsen, Imai, Wang, Sandoya, Kawecka-Jaszcz and Staessen. Drafting the manuscript: Sehestedt, Hansen, Torp-Pedersen and Staessen. Critical revision of the manuscript for important intellectual content: Sehestedt, Hansen, Li, Richart, Boggia, Kikuya, Thijs, Stolarz-Skrzypek, Casiglia, Tikhonoff, Malyutina, Nikitin, Björklund-Bodegård, Kuznetsova, Ohkubo, Lind, Torp-Pedersen, Jeppesen, Ibsen, Imai, Wang, Sandoya, Kawecka-Jaszcz and Staessen. Statistical analysis: Sehestedt, Hansen, Thijs and Staessen. Obtained funding: Staessen. Administrative, technical, or material support: Sehestedt, Hansen, Li, Richart, Boggia, Kikuya, Thijs, Stolarz-Skrzypek, Casiglia, Tikhonoff, Malyutina, Nikitin, Björklund-Bodegård, Kuznetsova, Ohkubo, Lind, Torp-Pedersen, Jeppesen, Ibsen, Imai, Wang, Sandoya, Kawecka-Jaszcz and Staessen. Study supervision: Staessen.

1 Clement DL, De Buyzere ML, De Bacquer DA, de Leeuw PW, Duprez DA, Fagard RH, Gheeraert PJ, Missault LH, Braun JJ, Six RO, Van der Niepen P, O'Brien E, for the Office versus Ambulatory Pressure Study investigators. Prognostic value of ambulatory bloodpressure recordings in patients with treated hypertension. N Engl J Med 2003; 348: 2407-2415.

2 Dolan E, Stanton A, Thijs L, Hinedi K, Atkins N, McClory S, Den Hond E, McCormack P, Staessen JA, O'Brien E. Superiority of ambulatory over clinic blood pressure measurement in predicting mortality. The Dublin Outcome Study. Hypertension 2005; 46: 156-161.

3 Hansen TW, Kikuya M, Thijs L, Björklund-Bodegård K, Kuznetsova T, Ohkubo T, Richart T, Torp-Pedersen C, Lind L, Jeppesen J, Ibsen H, Imai Y, Staessen JA, on behalf of the IDACO Investigators. Prognostic superiority of daytime ambulatory over conventional 
blood pressure in four populations: a meta-analasis of 7030 individuals. $J$ Hypertens 2007; 25: 1554-1564.

4 Stern N, Marcus Y. Hypertension in diabetes: the role of the vasculature. Curr Hypertens Rep 2004; 6: 90-97.

5 Cuspidi C, Meani S, Lonati L, Fusi V, Valerio C, Sala C, Magnaghi G, Maisaidi M, Zanchetti A. Short-term reproducibility of a non-dipping pattern in type 2 diabetic hypertensive patients. J Hypertens 2006; 24: 647-653.

6 Geiss LS, Rolka DB, Engelgau MM. Elevated blood pressure among US adults with diabetes, 1988-1994. Am J Prev Med 2002; 22: 42-48.

7 Maahs DM, Kinney GL, Wadwa P, Snell-Bergeon JK, Dabelea D, Hokanson J, Ehrlich J, Garg S, Eckel RH, Rewers MJ. Hypertension prevalence, awareness, treatment, and control in an adult type 1 diabetes population and a comparable general population. Diabetes Care 2005; 28: 301-306.

8 Eguchi K, Ishikawa J, Hoshide S, Pickering TG, Schwartz JE, Shimada K, Kario K. Nighttime blood pressure variability is a strong predictor for cardiovascular events in patients with Type 2 diabetes. Am J Hypertens 2009; 22: 46-51.

9 Kokubo $Y$, Okamura $T$, Watanabe $M$, Higashiyama A, Ono $Y$, Miyamoto $Y$, Furukawa $Y$, Kamide K, Kawanishi K, Okayama A, Yoshimasa Y. The combined impact of blood pressure category and glucose abnormality on the incidence of cardiovascular diseases in a Japanese urban cohort: the Suita Study. Hypertens Res 2010; 33: 1238-1243.

10 Tight blood pressure control and risk of macrovascular and microvascular complications in type 2 diabetes: UKPDS 38. UK Prospective Diabetes Study Group. Br Med J 1998; 317: 703-713.

11 Tuomilehto J, Rastenyte D, Birkenhager WH, Thijs L, Antikainen R, Bulpitt CJ, Fletche AE, Forette F, Goldhaber A, Palatini P, Sarti C, Fagard R. Effects of calcium-channel blockade in older patients with diabetes and systolic hypertension. Systolic hypertension in Europe trial investigators. N Engl J Med 1999; 340: 677-684.

12 Thijs L, Hansen TW, Kikuya M, Björklund-Bodegard K, Li Y, Dolan E, Tikhonoff V, Sleidlerová J, Kuznetsova T, Stolarz K, Bianchi M, Richart T, Casiglia E, Malyutina S, Filipovský J, Kawecka-Jaszcz K, Nikitin Y, Ohkubo T, Sandoya E, Wang JG, TorpPedersen $\mathrm{C}$, Lind L, Ibsen $\mathrm{H}$, Imai $\mathrm{Y}$, Staessen JA. The International Database of Ambulatory blood pressure in relation to Cardiovascular Outcome (IDACO): protocol and research perspectives. Blood Press Monit 2007; 12: 255-262.

13 Hansen TW, Jeppesen J, Rasmussen F, Ibsen H, Torp-Pedersen C. Ambulatory blood pressure monitoring and mortality: a population-based study. Hypertension 2005; 45: 499-504.

14 Staessen JA, Bieniaszewski L, O'Brien ET, Imai Y, Fagard R. An epidemiological approach to ambulatory blood pressure monitoring : the Belgian population study. Blood Press Monit 1996; 1: 13-26.

15 Ohkubo T, Hozawa A, Yamaguchi J, Kikuya M, Ohmori K, Michimata M, Matsubara M, Hashimoto J, Hoshi H, Araki T, Tsuji I, Satoh H, Hisamichi S, Imai Y. Prognostic significance of the nocturnal decline in blood pressure in individuals with and without high 24-h blood pressure: the Ohasama study. J Hypertens 2002; 20: 2183-2189.

16 Schettini C, Bianchi M, Nieto F, Sandoya E, Senra H, Hypertension Working Group. Ambulatory blood pressure. Normality and comparison with other measurements. Hypertension 1999; 34(Part 2): 818-825.

17 Li Y, Wang JG, Gao HF, Nawrot T, Wang GL, Qian YS, Staessen JA, Zhu DL. Are published characteristics of the ambulatory blood pressure generalizable to rural Chinese? The JingNing population study. Blood Press Monit 2005; 10: 125-134.

18 Ingelsson E, Björklund K, Lind L, Ärnlöv J, Sundström J. Diurnal blood pressure pattern and risk of congestive heart failure. JAMA 2006; 295: 2859-2866.

19 Kuznetsova T, Staessen JA, Kawecka-Jaszcz K, Babeanu S, Casiglia E, Filipovsk J, Nachev C, Nikitin Y, Pelesk J, O'Brien E, on behalf of the EPOGH Investigators. Quality control of the blood pressure phenotype in the European Project on Genes in Hypertension. Blood Press Monit 2002; 7: 215-224.

20 Kuznetsova T, Malyutina S, Pello E, Thijs L, Nikitin Y, Staessen JA. Ambulatory blood pressure of adults in Novosibirsk, Russia: interim report on a population study. Blood Press Monit 2000; 5: 291-296.

21 Imai Y, Nagai K, Sakuma M, Sakuma H, Nakatsuka H, Satoh H, Minami N, Munakata M, Hashimoto J, Yamagishi T, Watanabe N, Yabe T, Nishiyama A, Abe K. Ambulatory blood pressure of adults in Ohasama, Japan. Hypertension 1993; 22: 900-912.

22 O'Brien E, Mee F, Atkins N, Thomas M. Evaluation of three devices for self-measurement of blood pressure according to the revised British Hypertension Society Protocol: the Omron HEM-705 CP, Philips HP5332 and Nissei DS-175. Blood Press Monit 1996; 1: 55-62

23 Fagard R, Brguljan J, Thijs L, Staessen J. Prediction of the actual awake and asleep blood pressures by various methods of $24 \mathrm{~h}$ pressure analysis. J Hypertens 1996; 14: 557-563.

24 Mancia G, De Backer G, Dominiczak A, Cifkova R, Fagard R, Germano G, Grassi G, Heagerty AM, Kjeldsen SE, Laurent S, Narkiewicz K, Ruilope L, Rynkiewicz A, Schmieder RE, Struijker-Boudier HA, Zanchetti A, 2007 Guidelines for the management of arterial hypertension. The Task Force for the management of arterial hypertension of the European Society of Hypertension (ESH) and of the European Society of Cardiology (ESC). J Hypertens 2007; 25: 1105-1187.

25 Hansen TW, Jeppesen J, Rasmussen S, Ibsen H, Torp-Pedersen C. Ambulatory blood pressure and risk of cardiovascular disease: a population based study. Am J Hypertens 2006; 19: 243-259.
26 Nawrot TS, Van Hecke E, Thijs L, Richart T, Kuznetsova T, Jin Y, Vangronsveld J, Roels HA, Staessen JA. Cadmium-related mortality and long-term secular trends in the cadmium body burden of an environmetally exposed population. Environ Health Perspect 2008; 116: 1620-1628.

27 Tikhonoff V, Staessen JA, Kuznetsova T, Thijs L, Hasenkamp S, Bäumer V, Stolarz K, Seidlerová J, Filipovský J, Nikitin Y, Peleška J, Kawecka-Jaszcz K, Casiglia E, BrandHerrmann SM, Brand E, for the European Project on Genes in Hypertension (EPOGH) Investigators. SAH gene variants revisited in the European Project on Genes in Hypertension. J Hypertens 2008; 26: 244-250.

28 Easton DF, Peto J, Babiker AGAG. Floating absolute risk : an alternative to relative risk in survival and case-control analysis avoiding an arbitrary reference group. Stat Med 1991; 10: 1025-1035.

29 Eguchi K, Pickering TG, Hoshide S, Ishikawa J, Ishikawa S, Schwartz JE, Shimada K, Kario K. Ambulatory blood pressure is a better marker than clinic blood pressure in predicting cardiovascular events in patients with/without Type 2 diabetes. Am J Hypertens 2008; 21: 443-450.

30 Astrup AS, Nielsen FS, Rossing P, Ali S, Kastrup J, Smidt UM, Parving HH. Predictors of mortality in patients with type 2 diabetes with or without diabetic nephropathy: a follow-up study. J Hypertens 2007; 25: 2479-2485.

31 Mannucci E, Lambertucci L, Monami M, Fedeli A, Chiasserini V, Marchionni N, Masotti G, Ungar A. Pulse pressure and mortality in hypertensive type 2 diabetic patients. A cohort study. Diabetes Metab Res Rev 2006; 22: 172-175.

32 Palmas W, Pickering TG, Teresi J, Schwartz JE, Moran A, Weinstock RS, Shea S. Ambulatory blood pressure monitoring and all-cause mortality in elderly people with diabetes mellitus. Hypertension 2009; 53: 120-127.

33 Stamler J, Vaccaro O, Neaton JD, Wentworth D. Diabetes, other risk factors, and 12-yr cardiovascular mortality for men screened in the multiple risk factor intervention trial. Diabetes Care 1993; 16: 434-444.

34 Asia Pacific Cohort Studies Collaboration. Coronary risk prediction for those with and without diabetes. Eur J Cardiovasc Prev Rehabil 2006; 13: 30-36.

35 Iso H, Imano H, Kitamura A, Sato S, Naito Y, Tanigawa T, Ohira T, Yamagishi K, lida M, Shimamoto T. Type 2 diabetes and risk of non-embolic ischaemic stroke in Japanese men and women. Diabetologia 2004; 47: 2137-2144.

36 Adlerberth AM, Rosengren A, Wilhelmsen L. Diabetes and long-term risk of mortality from coronary and other causes in middle-aged Swedish men. A general population study. Diabetes Care 1998; 21: 539-545.

37 Stratton IM, Cull CA, Adler AI, Matthews DR, Neil HA, Holman RR. Additive effects of glycaemia and blood pressure exposure on risk of complications in type 2 diabetes: a prospective observational study (UKPDS 75). Diabetologia 2006; 49: 1761-1769.

38 Kannel WB, McGee DL. Diabetes and cardiovascular risk factors: the Framingham study. Circulation 1979; 59: 8-13.

$39 \mathrm{Gu} \mathrm{Q}$, Burt VL, Paulose-Ram R, Yoon S, Gillum RF. High blood pressure and cardiovascular disease mortality risk among US adults: the third National Health and Nutrition Examination Survey mortality follow-up study. Ann Epidemiol 2008; 18 : 302-309.

40 Hu G, Sarti C, Jousilahti P, Peltonen M, Qiao Q, Antikainen R, Tuomilehto J. The impact of history of hypertension and type 2 diabetes at baseline on the incidence of stroke and stroke mortality. Stroke 2005; 36: 2538-2543.

41 Conroy RM, Pyörälä K, Fitzgerald AP, Sans S, Menotti A, De Backer G, De Bacquer D, Ducimetière $P$, Jousilahti $P$, Keil $U$, Njølstad I, Oganov RG, Thomsen T, Tunstall-Pedoe H, Tverdal A, Wedel H, Whincup P, Wilhelmsen L, Graham $\mathrm{IM}$, on behalf of the SCORE project group. Estimates of 10-year risk of fatal cardiovascular disease in Europe: the SCORE project. Eur Heart J 2003; 24: 987-1003.

42 Wilson PWF, D'Agostino RB, Levy D, Belanger AM, Silbershatz $H$, Kannel WB. Prediction of coronary heart disease using risk factor categories. Circulation 1998; 97: $1837-1847$

43 Assmann G, Cullen P, Schulte H. Simple scoring scheme for calculating the risk of acute coronary events based on the 10-year follow-up of the prospective cardiovascular Munster (PROCAM) study. Circulation 2002; 105: 310-315.

44 Stevens RJ, Kothari V, Adler AI, Stratton IM. The UKPDS risk engine: a model for the risk of coronary heart disease in Type II diabetes (UKPDS 56). Clin Sci (Lond) 2001; 101: 671-679.

45 Guzder RN, Gatling W, Mullee MA, Mehta RL, Byrne CD. Prognostic value of the Framingham cardiovascular risk equation and the UKPDS risk engine for coronary heart disease in newly diagnosed Type 2 diabetes: results from a United Kingdom study. Diabet Med 2005; 22: 554-562.

46 Coleman RL, Stevens RJ, Retnakaran R, Holman RR. Framingham, SCORE, and DECODE risk equations do not provide reliable cardiovascular risk estimates in type 2 diabetes. Diabetes Care 2007; 30: 1292-1293.

47 Cushman WC, Evans GW, Byington RP, Goff Jr DC, Grimm Jr RH, Cutler JA, Simons-Morton DG, Basile JN, Corson MA, Probstfield JL, Katz L, Peterson KA, Friedewald WT, Buse JB, Bigger JT, Gerstein HC, Ismail-Beigi F. Effects of intensive blood-pressure control in type 2 diabetes mellitus. N Engl J Med 2010; 362: $1575-1585$

Supplementary Information accompanies the paper on Hypertension Research website (http://www.nature.com/hr) 